\title{
High expression of PRPS1 induces an anti-apoptotic effect in B-ALL cell lines and predicts an adverse prognosis in Chinese children with B-ALL
}

\author{
YIMEI MA ${ }^{1-3^{*}}$, XIZHOU AN ${ }^{1-3 *}$, XIANMIN GUAN ${ }^{1-3}$, QINGLIN KONG ${ }^{1-3}$, YANZHEN WANG $^{1-3}$, PENGFEI LI $^{1-3}$, \\ YAN MENG ${ }^{1-3}$, YINGHUI CUI ${ }^{1-3}$, XIANHAO WEN ${ }^{1-3}$, YUXIA GUO ${ }^{1-3}$, YALI SHEN ${ }^{1-3}$ and JIE YU ${ }^{1-3}$

\begin{abstract}
${ }^{1}$ Department of Hematology, Children's Hospital of Chongqing Medical University, Ministry of Education Key Laboratory of Child Development and Disorders; ${ }^{2}$ Chongqing Key Laboratory of Pediatrics; ${ }^{3}$ China International Science and Technology Cooperation Center for Child Development and Disorders, Chongqing Medical University, Chongqing 400014, P.R. China
\end{abstract}

Received November 11, 2016; Accepted November 16, 2017

DOI: $10.3892 / \mathrm{ol} .2018 .7903$

\begin{abstract}
Phosphoribosyl pyrophosphate synthetase 1 (PRPS1) is closely associated with a number of diseases; however, its influence in B-cell acute lymphoblastic leukemia (B-ALL) and the potential molecular mechanisms involved remain unclear. The present study aimed to evaluate the expression of PRPS1 in Chinese children with B-ALL and to investigate the mechanism of action of PRPS1 in this disease. A Cell Counting Kit-8 (CCK-8) assay was performed to examine the proliferation of B-ALL Sup-B15 and Raji cells, and flow cytometric analysis was conducted to determine the cell cycle distribution and rate of apoptosis. The mRNA and protein expression levels of PRPS1, MYC proto-oncogene, bHLH transcription factor, cyclin E1, B-cell lymphoma-2 (Bcl-2), cyclin dependent kinase 2 and caspase-3 were detected by reverse transcription-quantitative polymerase chain reaction and western blot analysis, respectively. Elevated PRPS1 expression was associated with a high-risk stratification and poor prognosis in patients with B-ALL. Furthermore, overexpression of PRPS1 accelerated the growth of and inhibited apoptosis in Sup-B15 and Raji cells as well as increasing
\end{abstract}

Correspondence to: $\mathrm{Dr} \mathrm{Jie} \mathrm{Yu}$, Department of Hematology, Children's Hospital of Chongqing Medical University, Ministry of Education Key Laboratory of Child Development and Disorders, Chongqing Medical University, 136 Zhongshan Second Road, Chongqing 400014, P.R. China

E-mail: 1808106657@qq.com

${ }^{*}$ Contributed equally

Abbreviations: AL, acute leukemia; B-ALL, B-cell acute lymphoblastic leukemia; T-ALL, T-cell lymphoblastic leukemia; BMMNC, bone marrow mono-nucleic cells; WBC, white blood cells; SR, standard risk; IR, intermediate risk; HR, high risk

Key words: B-cell acute lymphoblastic leukemia, phosphoribosyl pyrophosphate synthetase 1, B-cell lymphoma 2, apoptosis, prognosis the expression of Bcl-2 to induce an anti-apoptotic effect in B-ALL cell lines. The results of the present study indicate that PRPS1 regulates multiple processes in B-ALL and may be an attractive therapeutic target.

\section{Introduction}

Acute leukemia (AL) is the most common type of malignancy in children and adolescents; it includes B-cell acute lymphoblastic leukemia (B-ALL), T-cell ALL (T-ALL) and acute myeloid leukemia, and comprises $25-35 \%$ of all childhood cancer cases (1). The peak prevalence of AL incidence occurs between 2 and 5 years of age (1). B-ALL is the most common type of acute leukemia in children but, owing to risk stratification and improved supportive care, the survival rate of patients diagnosed with pediatric B-ALL has improved from 60 to $90 \%$ between 2000-2005 (2,3). However, the intensive and long-term cytotoxic treatment for B-ALL is inevitably accompanied by a large number of adverse effects. Identification of novel prognostic markers and therapeutic targets would assist in minimizing these effects by providing personalized treatment.

Aberrant purine synthesis leads to significant pathological defects in humans (4-8), thus the de novo purine biosynthesis pathway is a target for currently available cancer chemotherapy agents with an increasing number of novel agents continuously being developed. The phosphoribosyl pyrophosphate synthetase 1 gene (PRPS1), which encodes a rate-limiting purine biosynthesis enzyme, has been demonstrated to be associated with gouty arthritis, Arts syndrome, Charcot-Marie-Tooth disease 5 and hearing loss (9-14). However, a previous study indicated that PRPS1 also serves an important role in ALL relapse by reducing the feedback inhibition of de novo purine biosynthesis and competitive inhibition of thiopurine activation (15). All patients with ALL in the present study who harbored certain PRPS1 mutations relapsed early during treatment, and the number of ALL clones expressing this mutant protein exponentially expanded prior to clinical relapse.

To analyze the role of PRPS1 in pediatric B-cell acute lymphoblastic leukemia further, the expression of PRPS1 was 
measured in biopsy samples from children with B-ALL; it was revealed that elevated expression of PRPS1 was able to predict an adverse prognosis. Furthermore, the overexpression of PRPS1 in the B-ALL Sup-B15 and Raji cell lines induced B-cell lymphoma 2 (Bcl-2) overexpression, and a subsequent anti-apoptotic effect in these cells. These results indicate that PRPS1 is a potential therapeutic target for the treatment of B-ALL.

\section{Materials and methods}

Clinical samples and data. This study enrolled 71 pediatric patients who were newly diagnosed with B-ALL between March 2012 and November 2015 at the Children's Hospital of Chongqing Medical University (Chongqing, China). Among all patients, there were 42 males and 29 females with a median age of 50 months (4 years 2 months) and ranged from 8 months to 17 years and 2 months. The diagnosis of AL was performed using a standard French- American- British morphological analysis (16) and the morphology, immunology, cytogenetics and molecular biology criteria from the World Health Organization Classification of Tumors of Hematopoietic and Lymphoid tissues in 2008 (17), and the immunological phenotype was determined for all patients. For all patients enrolled in this study, the risk stratification and treatment options followed the Children's Cancer \& Leukemia Group-ALL2008 therapy guidelines (18). All the patients enrolled in this study were categorized into 3 risk groups: Standard risk, intermediate risk and high risk. They were categorized after the induction chemotherapy following the risk stratification standard in this guideline considering the risk factors at diagnosis, including peripheral white blood cell count, age, specific recurrent chromosomal abnormalities, and the response to initial therapy. There were 31 patients who achieved complete remission from AL and were enrolled as the remission group. Concurrently, 21 sex- and age-matched patients without a malignant hematological disorder were enrolled as a control group in the present study. The details of the included subjects are listed in Table I. The present study was approved by the Ethics Committee of Children's Hospital of Chongqing Medical University and followed all principles set by the Declaration of Helsinki. Informed consent was obtained from the parents or guardians of all participants. The basic information of all groups of patients is shown in Table I.

Bone marrow (BM) samples (1-2 $\mathrm{ml})$ of patients in each group were collected and treated with EDTA anticoagulant upon preliminary diagnosis, and following the achievement of complete remission. Mononuclear cells from the BM (BMMNC) were isolated by applying a density gradient centrifugation over Ficoll solution, later identified via light microscopy, and stored at $-80^{\circ} \mathrm{C}$ until further use.

Cell culture. The two human acute B-ALL cell lines (Sup-B15 and Raji, ATCC, Manassas, VA, USA) used in this study were obtained from the Ministry of Education Key Laboratory of Child Development and Disorders (Chongqing, China). Sup-B15 and Raji cells were cultured in RPMI-1640 supplemented with $10 \%$ heat-inactivated fetal bovine serum (both from Gibco; Thermo Fisher Scientific, Inc., Waltham, MA, USA) and $1 \mathrm{ml}$ glutamine, and were maintained at $37^{\circ} \mathrm{C}$ in a humidified atmosphere containing $5 \% \mathrm{CO}_{2}$. The cells were grown in suspension, the medium was changed every 2-3 days and the experiments were conducted with cells in the logarithmic growth phase.

Lentivirus infection. Lentivirus plasmids, used for the overexpression of PRPS1, and a negative control plasmid were purchased from Suzhou GenePharma Co., Ltd. (Suzhou, China). The cDNA sequence used for PRPS1 expression was matched to RefSeq NM_002764.3, from the National Center for Biotechnology Information GenBank (19). The viral titer was $\sim 1 \times 10^{9}$ transducing units in $1 \mathrm{ml}$ RPMI-1640 medium. Prior to infection, $3 \times 10^{6}$ Sup-B15 or Raji cells/ml were cultured in RPMI-1640 medium containing no serum. The cells were divided into two groups: The control group (infected with lentivirus produced from transfection with empty vector) and the PRPS1+ group (infected with lentivirus produced from transfection of the PRPS1-overexpressing vector). Once the cells were infected at multiplicity of infection of 3 , they were cultured at $37^{\circ} \mathrm{C}$ in $5 \% \mathrm{CO}_{2}$. The mRNA and protein expression levels of PRPS1 were identified by reverse transcription-quantitative polymerase chain reaction (RT-qPCR) and western blotting, respectively.

Cell counting Kit-8 (CCK-8) cell growth assay. Sup-B15 and Raji cells were cultured at a density of $5 \times 10^{3}$ cells/well in 96-well microtiter plates, and their proliferative abilities were evaluated at 0, 24, 48 and 96 h using CCK-8 (Nanjing KeyGen Biotech Co., Ltd., Nanjing, China), according to the manufacturer's protocol. Once the cells had been treated with CCK-8 at $37^{\circ} \mathrm{C}$ for $1 \mathrm{~h}$, the absorbance at $450 \mathrm{~nm}$ was measured in each well using a microplate reader. All assays were repeated three times in parallel.

Flow cytometry analysis of cell apoptosis. The apoptotic rate was measured via flow cytometry using a FACSCalibur flow cytometer (BD Biosciences, Franklin Lakes, NJ, USA) with the Annexin V-Allophycocyanin (APC)/7-aminoactinomycin (7AAD) Apoptosis kit (Nanjing KeyGen Biotech Co., Ltd., Nanjing China), following the manufacturer's protocol. The three aforementioned groups of Sup-B15 and Raji cells were harvested, and washed twice with ice-cold PBS. A total of $1 \times 10^{6}$ cells/condition were resuspended in $500 \mu 1$ $1 \mathrm{X}$ binding buffer, incubated with $5 \mu \mathrm{l}$ annexin $\mathrm{V}$-APC and $5 \mu 1$ 7AAD solution for $10 \mathrm{~min}$ at room temperature in the dark. The prepared samples were sorted using a flow cytometer and were analyzed by BD FACSDiva software v8.0.1 (BD Biosciences). All assays were repeated three times in parallel.

$R T-q P C R$. The expression of PRPS1 in BMMNCs from patients with either B-ALL or non-malignant hematological disease, as well as the expression of PRPS1, MYC proto-oncogene, bHLH transcription factor (c-Myc), cyclin E1, Bcl-2 and cyclin-dependent kinase 2 (CDK2) in Sup-B15 and Raji cells, were analyzed by RT-qPCR. Total RNA from $\sim 1 \times 10^{6}$ cells was extracted by using TRIzol (Invitrogen; Thermo Fisher Scientific, Inc.) following the manufacturer's protocol. Reverse transcription was performed using the 
PrimeScript ${ }^{\mathrm{TM}} \mathrm{RT}$ reagent kit. The primer sequences for PCR, designed based on cDNA sequence from GenBank database (https://www.ncbi.nlm.nih.gov/genbank/), are listed in Table II, with the $\beta$-actin gene used as an endogenous control. Quantitect SYBR ${ }^{\circledR}$ Green RT-PCR kit (Qiagen $\mathrm{GmbH}$, Hilden, Germany) was employed for the amplification reactions using the Applied Biosystems ${ }^{\circledR}$ StepOnePlus ${ }^{\mathrm{TM}}$ Real-time PCR system (Applied Biosystems; Thermo Fisher Scientific, Inc.) under following condition: $3 \mathrm{~min}$ pre-denaturation at $95^{\circ} \mathrm{C}$, followed by 40 cycles of denaturation at $95^{\circ} \mathrm{C}$ for $10 \mathrm{sec}$, annealing and extension at $60^{\circ} \mathrm{C}$ for $30 \mathrm{sec}$. The $\mathrm{Cq}$ value of each sample was obtained via the PCR system. The data were analyzed using the $2^{-\Delta \Delta C q}$ relative quantity algorithm (20). Each assay was repeated three times in parallel.

Western blot analysis. The antibodies used for western blotting, targeting PRPS1 (cat. no. ab137577), c-Myc (cat. no. ab32072), CDK2 (cat. no. ab32147), Bcl-2 (cat. no. ab32124), cyclin E1 (cat. no. ab33911) and caspase-3 (cat. no. ab32042), were purchased from Abcam (Cambridge, MA, USA) and the $\beta$-actin (cat. no. TA-09) antibody was acquired from Santa Cruz Biotechnology, Inc. (Dallas, TX, USA). All primary antibodies were diluted at a ratio of 1:1,000. Sup-B15 and Raji cells were harvested, and extracted using radioimmunoprecipitation assay buffer (Beyotime Institute of Biotechnology, Haimen, China), proteinase inhibitor cocktail (cat. no. P8340; Sigma-Aldrich; Merck KGaA, Darmstadt, Germany), PMSF (1 mM) and phosphatase inhibitor cocktail (cat. no. 04906845001; Roche Diagnostics GmbH, Mannheim, Germany). The protein concentration was measured using a bicinchoninic acid protein assay (Pierce; Thermo Fisher Scientific, Inc.). Approximately $100 \mathrm{ng}$ total protein/lane was separated on $8 \%$ SDS-PAGE gels and were transferred onto polyvinylidene fluoride membranes followed by blocking in $5 \%$ skimmed dry milk at room temperature for $1 \mathrm{~h}$. Next, the membranes were incubated with primary antibodies overnight at $4^{\circ} \mathrm{C}$. The membranes were rinsed three times with TBS-T (Tris-buffered saline with $0.1 \%$ Tween-20) and incubated with either horseradish peroxidase (HRP)-conjugated goat anti-rabbit (cat. no. ab6721; Abcam) or HRP-conjugated goat anti-mouse (cat. no. ab6789; Abcam) IgG (H+L) secondary antibody at a dilution of 1:2,000 at room temperature for $2 \mathrm{~h}$. The blots were visualized using a Clarity Max $^{\mathrm{TM}}$ Western ECL Substrate kit (cat. no. 1705062; Bio-Rad Laboratories, Inc., Hercules, CA, USA), according to the manufacturer's protocol and each assay was repeated three times in parallel. The amount of protein in each band was quantified using Quantity One 4.6.2 software (Bio-Rad Laboratories, Inc., Hercules, CA, USA).

Immunohistochemical (IHC) staining. BMMNCs smears were uniformly spread on poly-lysine coated slides at $2 \times 10^{5}$ cells/slide, and were fixed with $4 \%$ paraformaldehyde at room temperature for $20 \mathrm{~min}$ prior to being washed with PBS 3 times. The slides were incubated with $0.3 \%$ tritron- 100 at room temperature for $30 \mathrm{~min}$ to permeabilize the cells. Following washing with PBS for 3 times, the slides were blocked with $10 \%$ bovine serum albumin at room temperature for a further $30 \mathrm{~min}$. Slides were then incubated with a
Table I. Details of patient samples in the present study.

\begin{tabular}{lclc}
\hline Disease & Patients, no. & $\begin{array}{c}\text { Age, } \\
\text { years (range) }\end{array}$ & $\begin{array}{c}\text { Sex, } \\
\text { male/female }\end{array}$ \\
\hline B-ALL & 71 & $4.0(2.0-9.0)$ & $42 / 29$ \\
B-ALL-CR & 31 & $3.2(2.2-8.7)$ & $20 / 13$ \\
Control & 21 & $6.2(2.4-12-8)$ & $9 / 12$ \\
\hline
\end{tabular}

B-ALL, B-cell acute lymphoblastic leukemia; B-ALL-CR, B-ALL complete remission.

rabbit anti-human PRPS1 polyclonal antibody (dilution, 1:200; cat. no. ab137577; Abcam) at $4^{\circ} \mathrm{C}$ overnight followed by a peroxidase-conjugated goat anti-rabbit secondary antibody (dilution, 1:50; cat. no. TA140003; OriGene Technologies, Inc., Rockville, MD, USA) at room temperature for $30 \mathrm{~min}$. The slides were then stained with diaminobenzidine at room temperature for $2 \mathrm{~min}$ and counterstained with hematoxylin at room temperature for $40 \mathrm{sec}$. Images were acquired using a light microscope with Olympus BX51 system (Olympus Corporation, Tokyo, Japan) at x100 magnification.

Statistical analysis. Non-Gaussian numerical data were presented as the median with 25 th and 75 th quartiles, whereas either normally distributed data or data from parallel repeated experiments were presented as the mean \pm standard deviation. Statistical differences in each assay were analyzed via SPSS 20.0 (IBM Corp., Armonk, NY, USA). Significance was determined by applying an unpaired Student's t-test for Gaussian-distributed data from two groups, the Mann-Whitney test for data from two groups that did not follow Gaussian distribution and one-way analysis of variance, followed by the Bonferroni correction post hoc test, was used for data from more than two groups. $\mathrm{P}<0.05$ was considered to indicate a statistically significant difference.

\section{Results}

Expression of PRPS1 in pediatric patients with B-cell acute lymphoblastic leukemia. The expression of PRPS1, as determined by RT-qPCR, was significantly higher in patients newly diagnosed with B-ALL compared with those in the control group or those that had achieved complete remission (Fig. 1A and Table III).

IHC staining to detect the protein expression and distribution of PRPS1 in B-ALL and control patients revealed that the staining for PRPS1 in the BM was stronger in the B-ALL patients compared with that in the control group (Fig. 1B), which was consistent with the RT-qPCR data.

Increased PRPS1 expression is associated with high-risk stratification and poor prognosis in patients with $B-A L L$. The risk factors for B-ALL patients include an elevated peripheral white blood cell ( $\left.\mathrm{WBC} \geq 50 \times 10^{9} / 1\right)$ count, age (infant or $\geq 10$ years old), recurrent chromosomal abnormalities and poor response to initial therapy (2). Depending on these risk factors, the patients were categorized into 
Table II. List of primers for reverse transcription-quantitative polymerase chain reaction.

\begin{tabular}{|c|c|c|c|}
\hline Gene & Sequences & Template & Size, bp \\
\hline PRPS1 & & NM_001204402.1 & 127 \\
\hline Forward & 5'-GATGGCATAAACTCTGGTGGC-3' & & \\
\hline Reverse & 5'-GGTGCTTGTGGGAGATGTGAA-3' & & \\
\hline c-Myc & & NM_002467.4 & 180 \\
\hline Forward & 5'-CATTCTCTGCTCTCCTCGAC-3' & & \\
\hline Reverse & 5'-TCCAGACTCTGACCTTTGC-3' & & \\
\hline Cyclin E1 & & NM_001238.3 & 359 \\
\hline Forward & 5'-CTGGATGTTGACTGCCTTGA-3' & & \\
\hline Reverse & 5'-CCGCTGCTCTGCTTCTTAC-3' & & \\
\hline CDK2 & & NM_001798.4 & 395 \\
\hline Forward & 5'-CCTTGTTTGTCCCTTCTAC-3' & & \\
\hline Reverse & 5'-CAAATCCACCCACTATGA-3' & & \\
\hline $\mathrm{Bcl}-2$ & & NM_000633.2 & 119 \\
\hline Forward & 5'-CTGCACCTGACGCCCTTCACC-3' & & \\
\hline Reverse & 5'-CACATGACCCCACCGAACTCAAAGA-3' & & \\
\hline$\beta$-actin & & NM_001101.3 & 191 \\
\hline Forward & 5'-AAGATGACCCAGATCATGTTTGAGACC-3' & & \\
\hline Reverse & 5'-GCCAGGTCCAGACGCAGGAT-3' & & \\
\hline
\end{tabular}

PRPS1, phosphoribosyl pyrophosphate synthetase 1; c-Myc, MYC proto-oncogene, bHLH transcription factor; CDK2, cyclin-dependent kinase 2; Bcl-2, B-cell lymphoma-2.

the standard risk, intermediate risk and high-risk groups, according to the CCLG ALL2008 guidelines (18), and were treated with chemotherapy accordingly. Analysis of PRPS1 in these subgroups revealed a significant difference in PRPS1 levels between each of the three risk groups of patients with B-ALL (Fig. 2 and Table III), and PRPS1 expression was significantly increased in patients with higher risk stratification. These results indicated that PRPS1 expression was associated with risk stratification in B-ALL.

To understand the association of PRPS1 expression with the clinical manifestations of B-ALL patients further, the PRPS1 expression levels were compared with the clinical manifestations of children with B-ALL (Table III). The expression of PRPS1 was observed to be higher in samples from patients with a peripheral WBC count $>50 \times 10^{9} / 1$ and from patients with minimal residual disease. The expression level of PRPS1 was not associated with any other clinical manifestations in patients with B-ALL, including sex, age and the incidence of relapse. Although no significant difference in relapse was identified, patients who experienced relapse tended to have elevated PRPS1 expression levels $(\mathrm{P}=0.112)$ compared with those who did not experience relapse. Overall, these results indicated that increased expression of PRPS1 was able to predict a poor prognosis of children with B-ALL.

PRPS1 overexpression increases the growth and inhibits apoptosis of $B$-ALL cell lines. To investigate the function of PRPS1 in the leukemogenesis of B-ALL further, the B-ALL cell lines Sup-B15 and Raji were transfected with lentivirus particles containing the PRPS1-overexpressing vector. The increased PRPS1 expression was confirmed by RT-qPCR and western blot analysis to be significant in these cells $(\mathrm{P}<0.05$; Fig. 3$)$.

To examine the effect of PRPS1 overexpression on ALL cell behaviors, cell proliferation (Fig. 4A) and annexin V-APC/7AAD staining assays (Fig. 4B and C) were performed on Sup-B15 and Raji cells. The results of the cell proliferation assay revealed that the proliferation of cells overexpressing PRPS1 was significantly increased compared with cells that did not overexpress PRPS1 ( $<<0.05$; Fig. 4A), which indicated that PRPS1 overexpression significantly increased the proliferation of B-ALL cells. Furthermore, the results of the annexin $\mathrm{V}-\mathrm{APC} / 7 \mathrm{AAD}$ flow cytometry assay (Fig. 4B) demonstrated that, compared with the control groups, cells overexpressing PRPS1 exhibited a decrease in apoptosis $(\mathrm{P}<0.05$; Fig. $4 \mathrm{~B}$ and $\mathrm{C})$. The phase distributions of the cell cycle were detected; however, the results did not show any significant differences between the cells overexpressing PRPS1 and the control cells at any of the phases (Table IV). When accounting for the results in all assessed cell lines, PRPS1 overexpression decreased the apoptosis of B-ALL cell lines and thus inhibited the growth of cells, but this exogenous expression did not affect the distribution of cells in different phases of the cell cycle.

PRPS1 increases the expression of Bcl-2, inducing an anti-apoptotic effect in B-ALL cell lines. To investigate the anti-apoptotic effect induced by PRPS1 overexpression in B-ALL cell lines, the expression levels of c-Myc, cyclin E1, Bcl-2, CDK2 and caspase-3 were evaluated in Sup-B15, and Raji cells by RT-qPCR (Fig. 5A) and western blot analysis (Fig. 5B). The results revealed that $\mathrm{Bcl}-2$, which is a key 
Table III. Association of PRPS1 expression with clinical manifestations in AL.

\begin{tabular}{lccc}
\hline Characteristic & $\begin{array}{c}\text { Patients, } \\
\text { no. }\end{array}$ & $\begin{array}{c}\text { PRPS1 } \\
\text { expression }\end{array}$ & P-value \\
\hline $\begin{array}{l}\text { Sex } \\
\text { Male }\end{array}$ & 42 & $1.37(0.83-2.38)$ & 0.472 \\
$\quad$ Female & 29 & $1.05(0.66-2.58)$ & \\
Age, years & & & 0.124 \\
$\quad 1-10$ & 52 & $1.14(0.68-2.37)$ & \\
$\quad<1$ or $\geq 10$ & 19 & $1.67(0.89-2.67)$ & \\
WBC count & & & 0.020 \\
$\quad<50 x 10^{9} / 1$ & 47 & $0.99(0.69-1.73)$ & \\
$\quad 550 x 10^{9} / 1$ & 24 & $1.79(1.27-2.87)$ & \\
MRD & & & 0.026 \\
$\quad$ Positive & 8 & $3.69(1.03-4.750)$ & \\
$\quad$ Negative & 63 & $1.27(0.71-1.88)$ & \\
Relapse & & & 0.112 \\
$\quad$ Yes & 8 & $1.80(1.21-3.46)$ & \\
$\quad$ No & 63 & $1.27(0.71-2.37)$ & \\
Risk stratification & & & \\
SR group & 27 & $0.79(0.53-1.07)$ & $0.0017^{\mathrm{a}}$ \\
IR group & 21 & $1.28(0.91-2.11)$ & $0.0033^{\mathrm{b}}$ \\
HR group & 23 & $2.42(1.65-3.41)$ & $0.0000^{\mathrm{c}}$ \\
\hline
\end{tabular}

Data are expressed as the median (interquartile range) and are normalized to $\beta$-actin. ${ }^{\text {a }} \mathrm{SR}$ group compared with IR group; ${ }^{\mathrm{b}} \mathrm{IR}$ group compared with HR group; 'HR group compared with SR group. PRPS1, phosphoribosyl pyrophosphate synthetase 1; AL, acute leukemia; WBC, white blood cell; MRD, minimal residual disease; $\mathrm{SR}$, standard risk; IR, intermediate risk; HR, high risk.

inhibitor of the mitochondrial associated apoptosis pathway, was significantly upregulated $(\mathrm{P}<0.05)$ in $\mathrm{B}-\mathrm{ALL}$ cells overexpressing PRPS1. Protein expression of other apoptotic or cell cycle regulators, including CDK2, c-Myc, cyclin E1 and caspase-3, did not exhibit any significant changes in the assays in the two cell lines. These results indicate that Bcl-2 may be the effector that implements the anti-apoptotic function downstream of PRPS1.

\section{Discussion}

Evasion of apoptosis is an important mechanism for tumorigenesis (21), the death receptor pathway (22), the mitochondrial associated apoptosis pathway (23), and the crosstalk between these pathways (24) has been identified to serve important roles during apoptosis in cancer cells. The present study revealed that the purine synthetase PRPS1 was highly expressed in the BM samples of children suffering from B-ALL, and was able to induce an anti-apoptotic effect by increasing the expression of Bcl-2, which is an essential anti-apoptotic gene in the mitochondrial pathway (25). Compared with samples from the control group, PRPS1 was highly expressed in the samples from patients with B-ALL; furthermore, samples from

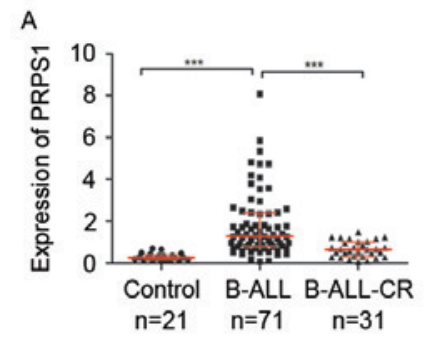

B
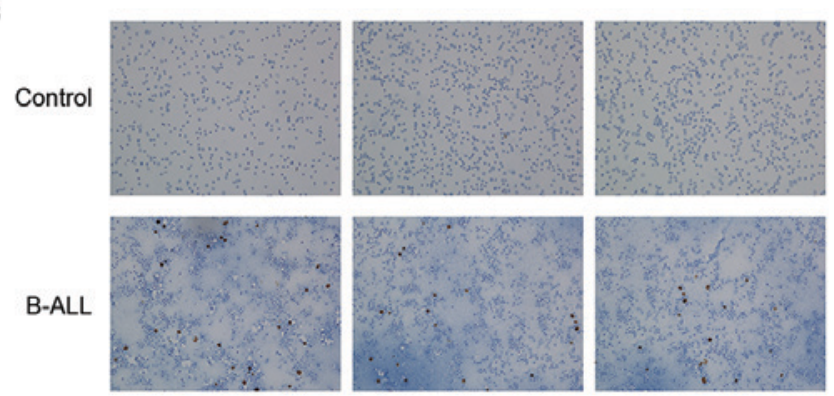

Figure 1. PRPS1 was highly expressed in samples from patients newly diagnosed with acute leukemia. (A) PRPS1 expression was detected by reverse transcription-quantitative polymerase chain reaction in different group of samples and was increased in B-ALL patients and was associated with disease progression and treatment status $\left({ }^{* * *} \mathrm{P}<0.001\right)$. (B) The representative figure of PRPS1 proteins in samples of B-ALL and control patients stained by immunohistochemical staining (magnification, x100). PRPS1, phosphoribosyl pyrophosphate synthetase 1; CR, complete remission; B-ALL, B-cell acute lymphoblastic leukemia.

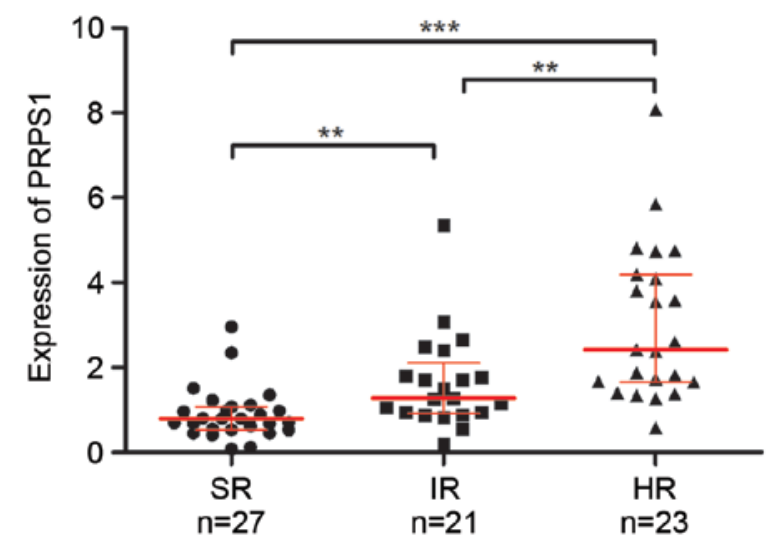

Figure 2. PRPS1 expression level was associated with risk stratification of B-ALL. PRPS1 expression was detected by reverse transcription-quantitative polymerase chain reaction in B-ALL patients grouped with diseases and risk stratification. ${ }^{* *} \mathrm{P}<0.01 ;{ }^{* * *} \mathrm{P}<0.001$. PRPS1, phosphoribosyl pyrophosphate synthetase 1; B-ALL, B-cell acute lymphoblastic leukemia SR, standard risk; $\mathrm{IR}$, intermediate risk; HR, high risk.

children newly diagnosed with B-ALL exhibited significantly higher expression levels of PRPS1 compared with patients who achieved complete remission. These findings indicate a likelihood of increased PRPS1 expression in B-ALL leukemic cells, but not in normal hematopoietic cells in this type of malignancy. Overexpression of PRPS1 in established B-ALL cell lines decreased the apoptotic rate in these cells, and a small but insignificant change was induced in other processes, including cell proliferation and phase distribution in the cell cycle. Determining changes in the expression of apoptotic genes revealed the increased expression of the key anti-apoptotic 
A

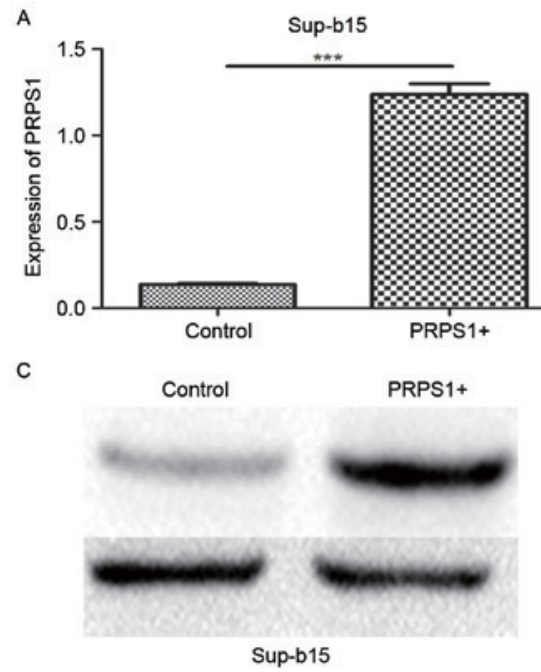

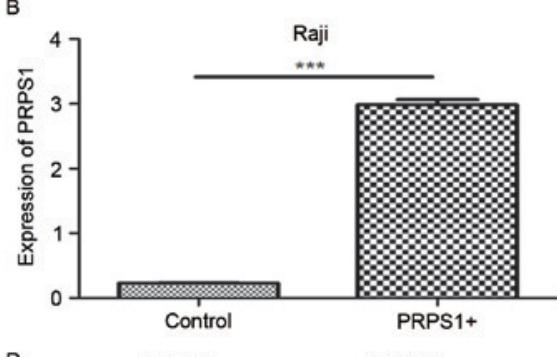

D

Control

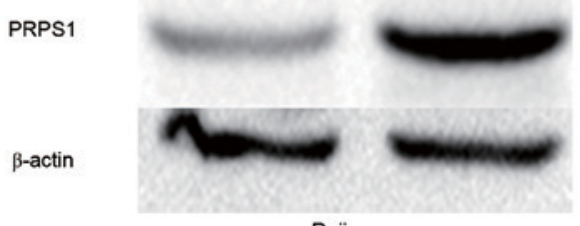

Raji

Figure 3. PRPS1 expression in Sup-b15 and Raji cells transfected with lentivirus. (A and B) PRPS1 mRNA levels detected by reverse transcription-quantitative polymerase chain reaction in the (A) Sup-b15 and (B) Raji cells infected with empty vector control and PRPS1-overexpressing lentivirus, respectively $\left({ }^{* * * *} \mathrm{P}<0.001\right)$. (C and D) PRPS1 protein levels detected by western blotting in (C) Sup-b15 and (D) Raji cells infected with empty vector control and PRPS1-overexpressing lentivirus, respectively. $\beta$-actin level was used as endogenous control. PRPS1+, cells overexpressing phosphoribosyl pyrophosphate synthetase 1.
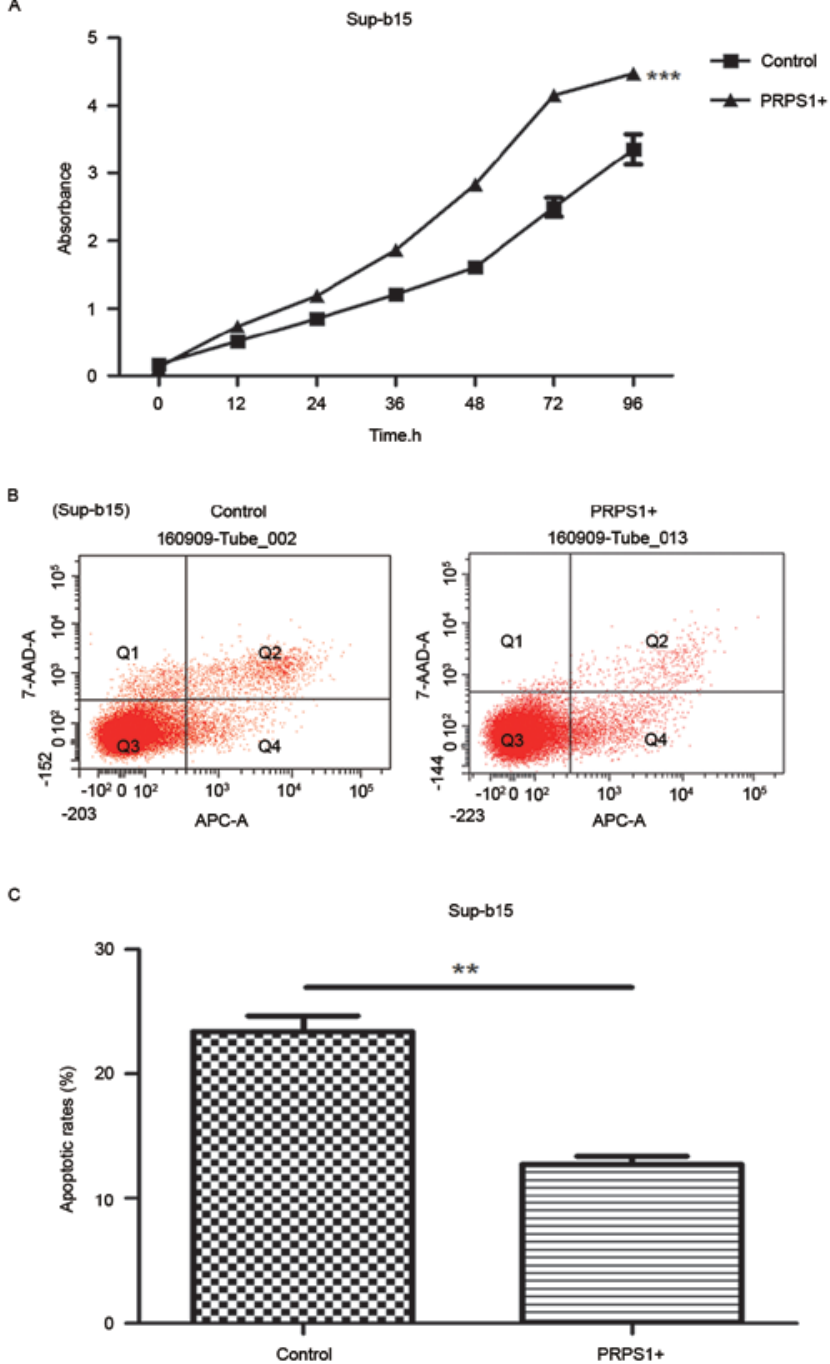
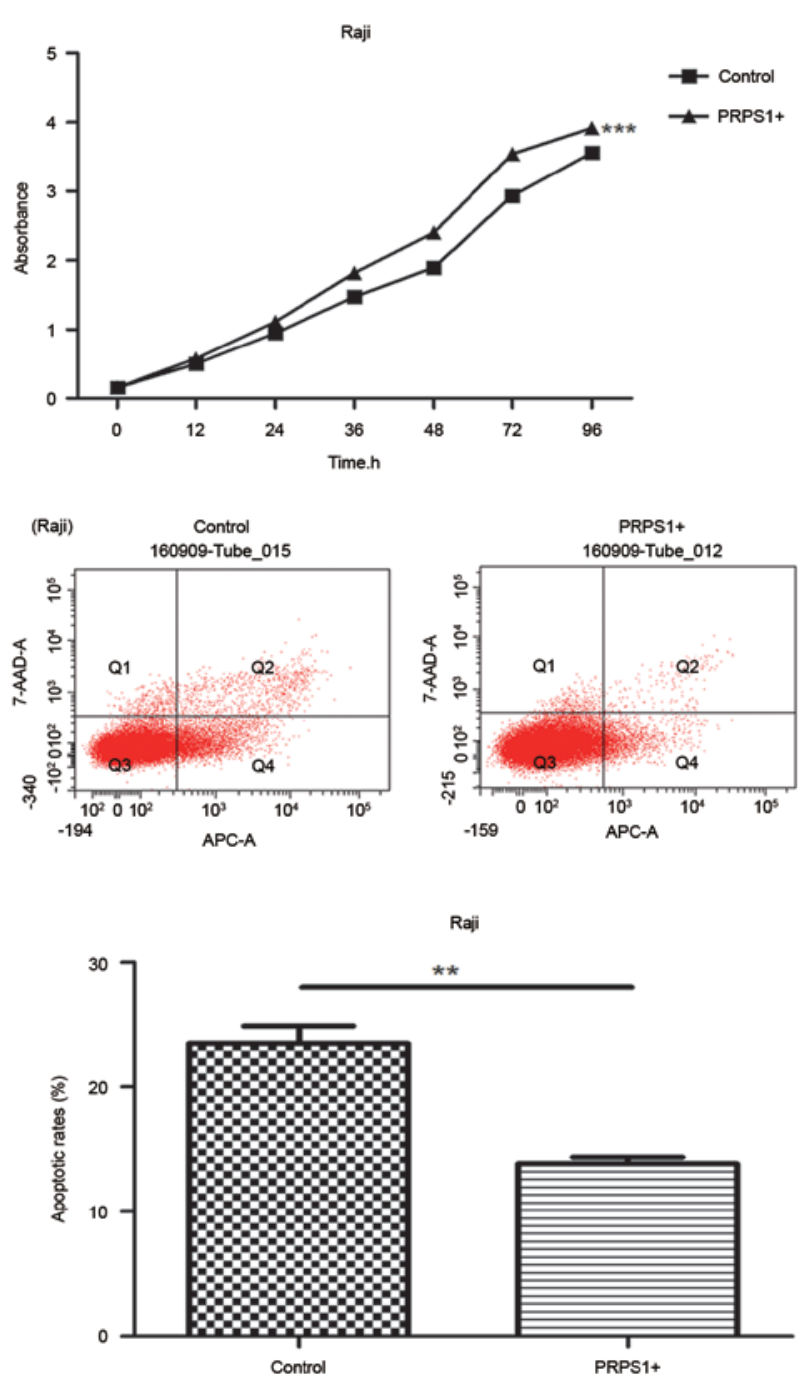

Figure 4. PRPS1 overexpression increased cell growth and decreased apoptosis of B-ALL cells. (A) PRPS1 overexpressing Sup-b15 and Raji cells exhibited increased cell growth compared with the vector control group. $\left({ }^{* * *} \mathrm{P}<0.001\right)$. (B) Flow cytometric cell counts of PRPS1 overexpressing and vector control Sup-b15 and Raji cells stained with annexin V-APC/7AAD. (C) Apoptotic rate of Sup-b15 and Raji cells overexpressing PRPS1 or not; PRPS1 overexpression significantly decreased the apoptosis of B-ALL cells. $\left.{ }^{(* *} \mathrm{P}<0.01\right)$. PRPS1+, cells overexpressing phosphoribosyl pyrophosphate synthetase 1 ; B-ALL, B-cell acute lymphoblastic leukemia; APC, allophycocynanin; 7AAD, 7-aminoactinomycin. 
Table IV. Cell cycle distribution in Sup-b15 and Raji cells.

\begin{tabular}{lcccccr}
\hline Cell line & $\mathrm{G}_{0} / \mathrm{G}_{1}$ phase & P-value & S phase & P-value & $\mathrm{G}_{2} / \mathrm{M}$ phase & P-value \\
\hline Sup-b15 & & 0.129 & & 0.086 & & 0.855 \\
PRPS1+ & $37.53 \pm 4.40$ & & $42.85 \pm 2.63$ & & $19.62 \pm 1.88$ & \\
VC & $43.30 \pm 3.98$ & & $37.36 \pm 3.79$ & & $19.34 \pm 1.85$ & \\
Raji & & 0.793 & & 0.464 & & 0.924 \\
PRPS1+ & $32.64 \pm 3.55$ & & $44.92 \pm 0.45$ & & $22.44 \pm 3.59$ & \\
VC & $32.04 \pm 1.09$ & & $45.77 \pm 1.75$ & & $22.19 \pm 2.09$ & \\
\hline
\end{tabular}

VC, vector control cells; PRPS1+, cells overexpressing phosphoribosyl pyrophosphate synthetase 1.

A
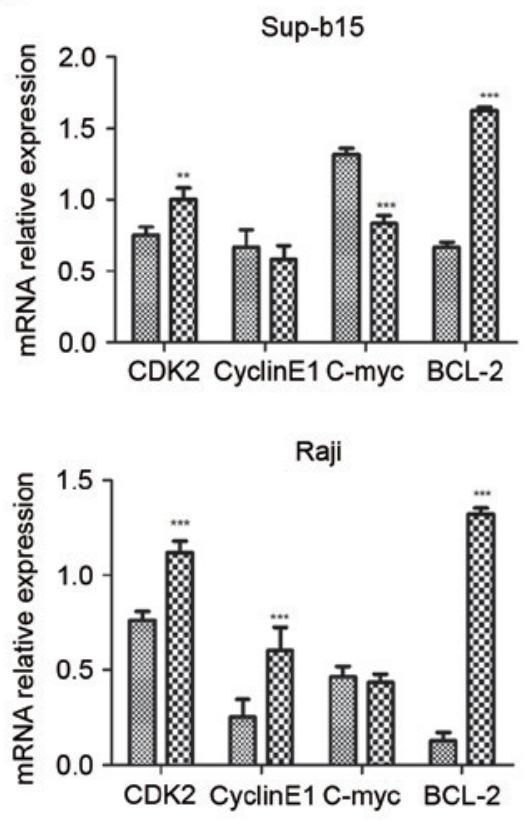

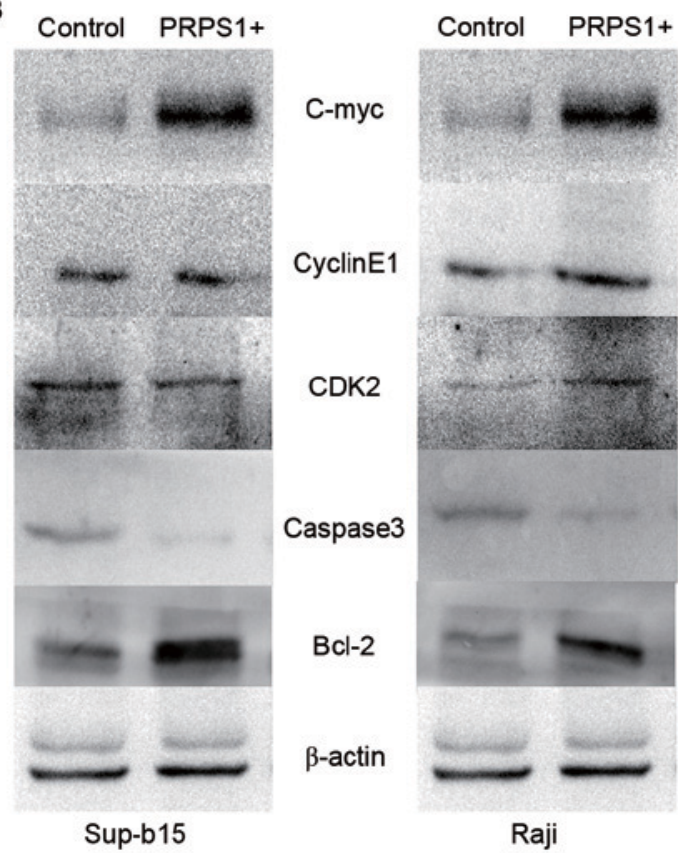

Figure 5. PRPS1 overexpression significantly increased the expression of Bcl-2. (A) mRNA expression of CDK2, cyclin E1, c-Myc and Bcl-2 detected by reverse transcription-quantitative polymerase chain reaction in PRPS1 overexpressing and vector control Sup-b15 and Raji cells; the mRNA expression of Bcl-2 was increased by PRPS1 overexpression. ( $\left.{ }^{* *} \mathrm{P}<0.01 ;{ }^{* * *} \mathrm{P}<0.001\right)$. (B) The protein expression of CDK2, Cyclin E1, c-Myc and Bcl-2 detected by western blotting in PRPS1 overexpressing and vector control Sup-b15 and Raji cells; the level of Bcl-2 protein was increased by PRPS1 overexpression. PRPS1+, cells overexpressing phosphoribosyl pyrophosphate synthetase 1; Bcl-2, B-cell lymphoma 2; c-Myc, MYC proto-oncogene, bHLH transcription factor; CDK2, cyclin-dependent kinase 2 .

gene Bcl-2 in cells overexpressing PRPS1. Multiple enzymes in the purine synthesis pathway are targets for nucleotide analog resistance inducers owing to their nucleotide metabolic activity; however, this novel function of PRPS1 provides another possibility in influencing tumorigenesis more directly by inhibiting apoptosis.

PRPS1 is a first-step enzyme that catalyzes the phosphoribosylation of ribose 5-phosphate to 5-phosphoribosyl-1-pyrophosphate, which is necessary in the de novo pathway for purine biosynthesis $(26,27)$. Defects in this gene are a cause of phosphoribosyl pyrophosphate synthetase superactivity, Charcot-Marie-Tooth disease X-linked recessive type 5 and Arts syndrome $(26,28)$. However, the role of PRPS1 in tumorigenesis has been seldom mentioned in the literature.

Recently, Li et al (15) identified PRPS1 mutations in pediatric B-ALL as specific relapse markers and identified mutated
PRPS1 as a driver for drug resistance. The present study overexpressed normal PRPS1 in B-ALL cell lines and investigated the function of unmutated PRPS1 during leukemogenesis. It was revealed that PRPS1 was able to increase the expression of $\mathrm{Bcl}-2$ and induce an anti-apoptotic effect in both B-ALL cell lines. These results indicate PRPS1 acts as an anti-apoptotic inducer in ALL and therefore, the results of the present study suggest a potential mechanism through which PRPS1 may affect the leukemic cells in the relapse process. Bcl-2, a key gene in the mitochondrial associated apoptosis pathway, is highly expressed in multiple types of malignancies and commonly induces the anti-apoptotic effect observed in tumorigenesis (29-32). The present study indicates that Bcl-2 is a downstream effector of PRPS1, owing to the response of its expression to the PRPS1 overexpression, which implies that PRPS1 exerts an anti-apoptotic. The Bcl-2-family inhibitor obatoclax has been identified 
as having potent cytotoxicity against MLL-rearranged and infant ALL cells in Phase III clinical trials (33). Furthermore, the results of the present study may indicate that Bcl-2 inhibitors represent a treatment option for pediatric patients with B-ALL who have increased levels of Bcl-2 or PRPS1.

Although the present study revealed a role of $\mathrm{Bcl}-2$ downstream of PRPS1, how PRPS1 regulates the expression of Bcl-2 remains unclear. As the elevated expression of PRPS1 was able to increase Bcl-2 expression at the RNA and protein level, PRPS1 may exert a transcriptional effect on Bcl-2, which indicates the possibility of PRPS1 functioning as a transcription factor to regulate Bcl-2. Kaida et al (34) identified PRPS1 as a P300-specific, but not a CREB-binding-protein binding protein in 293 cells, which indicates that PRPS1 possibly exerts transcription factor activities. Thus, further study of the regulatory mechanism of Bcl-2 expression by PRPS1 should be conducted.

The present study identified the purine synthetase PRPS1 as a leukemogenesis driver by increasing Bcl-2 expression and subsequently inducing an anti-apoptotic effect in B-ALL cells.

\section{Acknowledgements}

The present study was supported by funding from the Clinical Research Project of the Children's Hospital of Chongqing Medical University (grant no. lcyj2014-12) and the Medical Research Project of the Health and Family Planning Commission of Chongqing, China (grant nos. 2015MSXM042 and 2016ZDXM015).

\section{References}

1. Brisson GD, Alves LR and Pombo-de-Oliveira MS: Genetic susceptibility in childhood acute leukaemias: A systematic review. Ecancermedicalscience 9: 539, 2015.

2. Inaba H, Greaves M and Mullighan CG: Acute lymphoblastic leukaemia. Lancet 381: 1943-1955, 2013.

3. Hunger SP, Lu X, Devidas M, Camitta BM, Gaynon PS, Winick NJ, Reaman GH and Carroll WL: Improved survival for children and adolescents with acute lymphoblastic leukemia between 1990 and 2005: A report from the children's oncology group. J Clin Oncol 30: 1663-1669, 2012.

4. Zhang F, Patel DM, Colavita K, Rodionova I, Buckley B, Scott DA, Kumar A, Shabalina SA, Saha S, Chernov M, et al: Arginylation regulates purine nucleotide biosynthesis by enhancing the activity of phosphoribosyl pyrophosphate synthase. Nature Commun 6: 7517, 2015.

5. Turner RN, Aherne GW and Curtin NJ: Selective potentiation of lometrexol growth inhibition by dipyridamole through cell-specific inhibition of hypoxanthine salvage. $\mathrm{Br} \mathrm{J}$ Cancer 76 : 1300-1307, 1997.

6. Sessa C, de Jong J, D'Incalci M, Hatty S, Pagani O and Cavalli F: Phase I study of the antipurine antifolate lometrexol (DDATHF) with folinic acid rescue. Clin Cancer Res 2: $1123-1127,1996$

7. Laohavinij S, Wedge SR, Lind MJ, Bailey N, Humphreys A, Proctor M, Chapman F, Simmons D, Oakley A, Robson L, et al: A phase I clinical study of the antipurine antifolate lometrexol (DDATHF) given with oral folic acid. Invest New Drugs 14: 325-335, 1996.

8. Wedge SR, Laohavinij S, Taylor GA, Boddy A, Calvert AH and Newell DR: Clinical pharmacokinetics of the antipurine antifolate (6R)-5,10- dideaza-5,6,7,8-tetrahydrofolic acid (Lometrexol) administered with an oral folic acid supplement. Clin Cancer Res 1: 1479-1486, 1995.

9. Pei W, Xu L, Varshney GK, Carrington B, Bishop K, Jones M, Huang SC, Idol J, Pretorius PR, Beirl A, et al: Additive reductions in zebrafish PRPS1 activity result in a spectrum of deficiencies modeling several human PRPS1-associated diseases. Sci Rep 6: 29946, 2016
10. Maruyama K, Ogaya S, Kurahashi N, Umemura A, Yamada K, Hashiguchi A, Takashima H, Torres RJ and Aso K: Arts syndrome with a novel missense mutation in the PRPS1 gene: A case report. Brain Dev 38: 954-958, 2016.

11. Mittal R, Patel K, Mittal J, Chan B, Yan D, Grati M and Liu XZ: Association of PRPS1 mutations with disease phenotypes. Dis Markers 2015: 127013, 2015.

12. Gandia M, Fernández-Toral J, Solanellas J, Domínguez-Ruiz M, Gómez-Rosas E, Del Castillo FJ, Villamar M, Moreno-Pelayo MA and Del Castillo I: Mutations in PRPS1 causing syndromic or nonsyndromic hearing impairment: Intrafamilial phenotypic variation complicates genetic counseling. Pediatr Res 78: 97-102, 2015.

13. Almoguera B, He S, Corton M, Fernandez-San Jose P, Blanco-Kelly F, López-Molina MI, García-Sandoval B, Del Val J, Guo Y, Tian L, Liu X, et al: Expanding the phenotype of PRPS1 syndromes in females: neuropathy, hearing loss and retinopathy. Orphanet J Rare Dis 9: 190, 2014.

14. Robusto M, Fang M, Asselta R, Castorina P, Previtali SC, Caccia S, Benzoni E, De Cristofaro R, Yu C, Cesarani A, et al: The expanding spectrum of PRPS1-associated phenotypes: Three novel mutations segregating with X-linked hearing loss and mild peripheral neuropathy. Eur J Hum Genet 23: 766-773, 2015.

15. Li B, Li H, Bai Y, Kirschner-Schwabe R, Yang JJ, Chen Y, Lu G, Tzoneva G, Ma X, Wu T, et al: Negative feedback-defective PRPS1 mutants drive thiopurine resistance in relapsed childhood ALL. Nat Med 21: 563-571, 2015.

16. Hassan K, Bukhari KP, Zafar A, Malik MZ and Akhtar MJ: Acute leukaemia in children-French-American-British (FAB) classification and its relation to clinical features. J Pak Med Assoc 42: 29-31, 1992.

17. Vardiman JW, Thiele J, Arber DA, Brunning RD, Borowitz MJ, Porwit A, Harris NL, Le Beau MM, Hellström-Lindberg E, Tefferi A and Bloomfield CD: The 2008 revision of the world health organization (WHO) classification of myeloid neoplasms and acute leukemia: Rationale and important changes. Blood 114: 937-951, 2009.

18. Cui L, Gao C, Zhang RD, Jiao Y, Li WJ, Zhao XX, Liu SG, Yue ZX, Zheng HY, Deng GR, et al: Low expressions of ARS2 and CASP8AP2 predict relapse and poor prognosis in pediatric acute lymphoblastic leukemia patients treated on China CCLG-ALL 2008 protocol. Leuk Res 39: 115-123, 2015.

19. He M, Chao L and You YP: PRPS1 silencing reverses cisplatin resistance in human breast cancer cells. Biochem Cell Biol 95: 385-393, 2017.

20. Livak KJ and Schmittgen TD: Analysis of relative gene expression data using real-time quantitative PCR and the 2(-Delta Delta C(T)) method. Methods 25: 402-408, 2001.

21. Hanahan D and Weinberg RA: Hallmarks of cancer: The next generation. Cell 144: 646-674, 2011

22. Peter ME, Hadji A, Murmann AE, Brockway S, Putzbach W, Pattanayak A and Ceppi P: The role of CD95 and CD95 ligand in cancer. Cell Death Differ 22: 885-886, 2015.

23. Lopez J and Tait SW: Mitochondrial apoptosis: Killing cancer using the enemy within. Br J Cancer 112: 957-962, 2015.

24. Ola MS, Nawaz M and Ahsan $\mathrm{H}$ : Role of Bcl-2 family proteins and caspases in the regulation of apoptosis. Mol Cell Biochem 351: 41-58, 2011

25. Murphy KM, Ranganathan V, Farnsworth ML, Kavallaris M and Lock RB: Bcl-2 inhibits Bax translocation from cytosol to mitochondria during drug-induced apoptosis of human tumor cells. Cell Death Differ 7: 102-111, 2000.

26. Becker MA: Phosphoribosylpyrophosphate synthetase and the regulation of phosphoribosylpyrophosphate production in human cells. Prog Nucleic Acid Res Mol Biol 69: 115-148, 2001.

27. Yamada Y, Yamada K, Nomura N, Yamano A,Kimura R, Naiki M, Fukushi D, Wakamatsu N, Taniguchi A, Yamaoka N, et al: Molecular analysis of X-linked inborn errors of purine metabolism: HPRT1 and PRPS1 mutations. Nucleosides Nucleotides Nucleic Acids 30: 1272-1275, 2011

28. de Brouwer AP, van Bokhoven H, Nabuurs SB, Arts WF, Christodoulou J and Duley J: PRPS1 mutations: four distinct syndromes and potential treatment. Am J Hum Genet 86: 506-518, 2010.

29. Kim S, Nam SJ, Kwon D, Kim H, Lee E, Kim TM, Heo DS, Park SH, Kim CW and Jeon YK: MYC and BCL2 overexpression is associated with a higher class of memorial sloan-kettering cancer center prognostic model and poor clinical outcome in primary diffuse large B-cell lymphoma of the central nervous system. BMC Cancer 16: 363, 2016. 
30. Cabrera Ortega AA, Gonçalves Vde P, Guimarães MR, Rossa Junior C and Spolidorio LC: Overexpression of Bcl-2, SOCS 1, 3 and Cdh 1,2 are associated with the early neoplasic changes in modified 4-nitroquinoline 1-oxide-induced murine oral cancer model. J Oral Pathol Med 45: 573-580, 2016.

31. Jager R, Herzer U, Schenkel J and Weiher H: Overexpression of Bcl-2 inhibits alveolar cell apoptosis during involution and accelerates c-myc-induced tumorigenesis of the mammary gland in transgenic mice. Oncogene 15: 1787-1795, 1997.

32. Hsu SY, Lai RJ, Finegold M and Hsueh AJ: Targeted overexpression of Bcl-2 in ovaries of transgenic mice leads to decreased follicle apoptosis, enhanced folliculogenesis, and increased germ cell tumorigenesis. Endocrinology 137: 4837-4843, 1996.
33. Urtishak KA, Edwards AY, Wang LS, Hudome A, Robinson BW, Barrett JS, Cao K, Cory L, Moore JS, Bantly AD, et al: Potent obatoclax cytotoxicity and activation of triple death mode killing across infant acute lymphoblastic leukemia. Blood 121: 2689-2703, 2013.

34. Kaida A, Ariumi Y, Baba K, Matsubae M, Takao T and Shimotohno K: Identification of a novel p300-specific-associating protein, PRS1 (phosphoribosylpyrophosphate synthetase subunit 1). Biochem J 391: 239-247, 2005.

(c) (i) () $\odot$ This work is licensed under a Creative Commons EY NG No Attribution-NonCommercial-NoDerivatives 4.0 International (CC BY-NC-ND 4.0) License. 